\title{
Observational uncertainty and regional climate model evaluation: A pan-European perspective
}

Sven Kotlarski (1), Péter Szabó (2), Sixto Herrera (3), Olle Räty (4), Klaus Keuler (5), Pedro M. Soares (6), Rita M. Cardoso (6), Thomas Bosshard (7), Christian Pagé (8), Fredrik Boberg (9), José M. Gutiérrez (3), Adam Jaczewski (10), Frank Kreienkamp (11), Mark. A. Liniger (1), Cristian Lussana (12), and Gabriella Szepszo (2)

(1) Federal Office of Meteorology and Climatology MeteoSwiss, Switzerland, (2) Hungarian Meteorological Service, Hungary, (3) University of Cantabria, Spain, (4) University of Helsinki, Finland, (5) Brandenburg University of Technology, Germany, (6) Instituto Dom Luiz, Portugal, (7) Swedish Meteorological and Hydrological Institute, Sweden, (8) Centre Européen de Recherche et de Formation Avancée en Calcul Scientifique, France, (9) Danish Meteorological Institute, Denmark, (10) Institute of Meteorology and Water Management, Poland, (11) German Weather Service, Germany, (12) Norwegian Meteorological Institute, Norway

Local and regional climate change assessments based on downscaling methods crucially depend on the existence of accurate and reliable observational reference data. In dynamical downscaling via regional climate models (RCMs) observational data can influence model development itself and, later on, model evaluation, parameter calibration and added value assessment. In empirical-statistical downscaling, observations serve as predictand data and directly influence model calibration with corresponding effects on downscaled climate change projections.

Focusing on the evaluation of RCMs, we here analyze the influence of uncertainties in observational reference data on evaluation results in a well-defined performance assessment framework and on a European scale. For this purpose we employ three different gridded observational reference grids, namely (1) the well-established EOBS dataset (2) the recently developed EURO4M-MESAN regional re-analysis, and (3) several national high-resolution and quality-controlled gridded datasets that recently became available. In terms of climate models five reanalysis-driven experiments carried out by five different RCMs within the EURO-CORDEX framework are used. Two variables (temperature and precipitation) and a range of evaluation metrics that reflect different aspects of RCM performance are considered. We furthermore include an illustrative model ranking exercise and relate observational spread to RCM spread.

The results obtained indicate a varying influence of observational uncertainty on model evaluation depending on the variable, the season, the region and the specific performance metric considered. Over most parts of the continent, the influence of the choice of the reference dataset for temperature is rather small for seasonal mean values and inter-annual variability. Here, model uncertainty (as measured by the spread between the five RCM simulations considered) is typically much larger than reference data uncertainty. For parameters of the daily temperature distribution and for the spatial pattern correlation, however, important dependencies on the reference dataset can arise. The related evaluation uncertainties can be as large or even larger than model uncertainty. For precipitation the influence of observational uncertainty is, in general, larger than for temperature. It often dominates model uncertainty especially for the evaluation of the wet day frequency, the spatial correlation and the shape and location of the distribution of daily values. But even the evaluation of large-scale seasonal mean values can be considerably affected by the choice of the reference. When employing a simple and illustrative model ranking scheme on these results it is found that RCM ranking in many cases depends on the reference dataset employed. 